\title{
miR-187 induces apoptosis of SiHa cervical carcinoma cells by downregulating Bcl-2
}

\author{
C.Y. He ${ }^{1,2}$ and J. Yang ${ }^{1}$ \\ ${ }^{1}$ Department of Obstetrics and Gynecology, \\ Renmin Hospital of Wuhan University, Wuhan, Hubei Province, China \\ ${ }^{2}$ Central Hospital of Wuhan University, Enshi Autonomous Prefecture, \\ Enshi City, Hubei Province, China \\ Corresponding author: J. Yang \\ E-mail: jingyangzxc@sina.com
}

Genet. Mol. Res. 16 (1): gmr16018969

Received July 14, 2016

Accepted October 11, 2016

Published January 23, 2017

DOI http://dx.doi.org/10.4238/gmr16018969

Copyright (C) 2017 The Authors. This is an open-access article distributed under the terms of the Creative Commons Attribution ShareAlike (CC BY-SA) 4.0 License.

\begin{abstract}
Cervical carcinoma is a life-threatening illness posing considerable danger to women's health. microRNAs (miRNAs) have been shown to regulate multiple cellular events, including growth and proliferation, and miR-187 is thought to regulate the growth and apoptosis of certain cell types. Our study focused on the influence of miR-187 on the growth, proliferation, and apoptosis of SiHa cervical carcinoma cells, and explored the mechanism behind its pro-apoptotic effect. miR-187 and control (scrambled) miRNA were synthesized with a standard protocol and lipofected into $\mathrm{SiHa}$ cells. Thiazolyl blue tetrazolium bromide assays and tests of caspase-3 activity were then performed to examine growth, proliferation, and apoptosis by flow cytometry. Small interfering RNA (siRNA) and an expression plasmid were synthesized for inhibition and overexpression of Bcl-2, respectively, and following their transfection, western blotting was used to examine Bcl-2 protein levels. Compared to transfection with control
\end{abstract}


miRNA, miR-187 significantly reduced $\mathrm{SiHa}$ cell growth and decreased Bcl-2 expression. Increased translocation of phosphatidylserine and activation of caspase-3 were observed in miR-187-transfected cells. Moreover, inhibition of Bcl-2 enhanced the pro-apoptotic effect of this miRNA, while Bcl-2 overexpression had the opposite effect. miR-187 inhibits the growth and proliferation of $\mathrm{SiHa}$ cells, and induces their apoptosis via downregulation of $\mathrm{Bcl}-2$. Bcl-2 represents a potential therapeutic target for cervical carcinoma.

Key words: miR-187; Bcl-2; Cervical cancer; Apoptosis

\section{INTRODUCTION}

Cervical carcinoma is a malignancy of the female genital system with a relatively high mortality rate of approximately $12 \%$ (Chang et al., 2014). Despite its complicated pathogenesis, recent studies have demonstrated that viral infection, sexual behavior, and number of births are major cervical carcinoma determinants (Yuan et al., 2014). Recent clinical trials have shown that cervical carcinoma represents a significant threat to women's health (PedrozaTorres et al., 2014; Wen et al., 2015).

Early detection and treatment are the principle strategies of cervical carcinoma therapy. Although chemotherapy, radiotherapy, and surgery have been used to treat this disease with promising efficacy, certain disadvantages impede their further development, including clinical complications such as bleeding and toxic side effects (Huang et al., 2013; Zhang et al., 2013; Yu et al., 2014). Thus, the current emphasis and challenge in cervical carcinoma clinical practice is the improvement of treatment efficacy.

A precision medicine approach has recently been considered for cervical carcinoma treatment, with specific therapies being explored (Liu et al., 2013; Chen et al., 2014; Yang et al., 2014). This strategy includes the identification of more accurate targeting molecules (Luo et al., 2013), the efficacies of which need to be improved to enhance the effectiveness of cervical carcinoma therapy (Luo et al., 2013; Phuah et al., 2013). microRNAs (miRNAs) show promise as novel therapeutic targets for the treatment of this malignancy (Phuah et al., 2013).

miRNAs represent a category of short, non-coding RNAs with multiple biological functions. For example, miR-218 has been shown to inhibit the growth of cervical carcinoma cells, while miR-34a is associated with tumor metastasis (Xu et al., 2013; Li et al., 2015; Cong et al., 2016). This suggests that miRNAs are involved in the occurrence and development of cervical carcinoma (Cong et al., 2016). In addition, preliminary experiments have demonstrated that in cancer cells, expression of miR-187 is significantly higher than that in normal tissues (Bonetta et al., 2015; Song et al., 2015). The present study explored the regulatory effect of miR-187 on the SiHa cervical carcinoma cell line.

Apoptosis is a form of programmed cell death regulated by several proteins with antiand pro-apoptotic effects. Anti-tumor strategies aim to kill cancer cells while leaving normal cells undamaged. One potential mechanism to achieve this involves inducing apoptosis by regulating the proteins that promote and inhibit this process (Bumrungthai et al., 2015; Kong et al., 2015). Bcl-2 is an extensively studied inhibitor of apoptosis, and as such, drugs targeting it have been designed. However, the efficacy of such molecules in inhibiting Bcl-2 is limited (Deng et al., 2015; Jia et al., 2016). Furthermore, recent research has demonstrated that Bcl-2

Genetics and Molecular Research 16 (1): gmr16018969 
is regulated by multiple miRNAs (Liu et al., 2015). Our study aimed to establish an effective way to decrease Bcl-2 levels using miR-187.

In summary, the current investigation focused on the influence of miR-187 on the growth, proliferation, and apoptosis of SiHa cervical carcinoma cells, and explored the mechanism by which it induces apoptosis via Bcl-2.

\section{MATERIAL AND METHODS}

\section{Reagents and cell model}

Fetal bovine serum and Dulbecco's modified Eagle's medium (DMEM) were purchased from Hualan Biological Engineering, Inc. (Beijing, China). The following sequences were synthesized for RNA interference experiments: miR-187, 5'-ATC ACT GCA ATC GTC AGG TCA T-3' and 5'-AAG AAC GGT AAG AAG TAA GCA-3'; control miRNA, 5'-CCT GAG GGT TCA ACT CTA GC-3' and 5'-TTA CGA TTG TCA CGT ACA T-3'; Bcl-2 small interfering RNA (siRNA), 5'-GCT ACA GTC CAC TAT GGT GC-3' and 5'-TAC GAT TCG ATC AGT ACT T-3'.

The following materials were used in this study: Bcl-2 overexpression plasmid (Suzhou GenePharma, Suzhou, China), liposome transfection kit (Lipofectamine 2000; Invitrogen, Carlsbad, CA, USA), thiazolyl blue tetrazolium bromide (MTT) assay kit (Beijing Dingguo Biotechnology, Beijing, China), annexin V-fluorescein isothiocyanate (FITC) and caspase-3 kits (Beyotime, Haimen, China), and anti-Bcl-2 (catalog No.: N-19) and anti-actin antibodies (catalog No.: I-19) (Santa Cruz, Dallas, TX, USA).

$\mathrm{SiHa}$ cervical carcinoma cells were purchased from the American Type Culture Collection (Manassas, VA, USA). The following treatment groups were used: control miRNA; miR-187; control miRNA+Bcl-2 siRNA plasmid, miR-187+Bcl-2 siRNA; and control miRNA+Bcl-2 overexpression plasmid, miR-187+Bcl-2 overexpression plasmid.

\section{Cell culture}

SiHa cells were revived and cultured in high-glucose DMEM according to a standard protocol (Chen et al., 2014).

\section{Transfection}

Separately, miR-187 and control miRNA were transfected into SiHa cells using routine methods (Chen et al., 2014) and Lipofectamine 2000. Cells were cultured until $80 \%$ confluent, then miR-187 or control microRNA at a final concentration of $10 \mathrm{nM}$ was suspended in Lipofectamine 2000 and transfected into cells.

\section{MTT assay}

The MTT assay was performed following a previously published protocol (Liu et al., 2013). Specifically, $1 \times 10^{6} \mathrm{SiHa}$ cells were seeded in each well of a six-well plate and cultured for $8 \mathrm{~h}$. MTT $(2 \mathrm{mg} / \mathrm{mL})$ was added to each well and incubated with cells for $4 \mathrm{~h}$. Cells were then exposed to DMSO for $5 \mathrm{~min}$, before absorption at $560 \mathrm{~nm}$ was tested on a

Genetics and Molecular Research 16 (1): gmr16018969 
microplate reader. Optical density was plotted on the ordinate, with different groups on the abscissa, to assess the effect of treatments on cell proliferation. Untransfected cells and miR187-transfected cells were treated as negative and positive controls, respectively.

\section{Flow cytometry}

Flow cytometry was performed to examine apoptosis with the annexin V-FITC staining method (Li et al., 2015). Excitation and absorption wavelengths of 488 and $625 \mathrm{~nm}$, respectively, were used. Cells were stained with annexin V-FITC for $20 \mathrm{~min}$. The untransfected and miR-187-transfected groups were treated as negative and positive controls, respectively. All experiments were repeated three times.

\section{Western blot}

Proteins were extracted from transfected cells for western blotting according to standard methods (Cong et al., 2016). Extraction was carried out by lysing $1 \times 10^{5}$ cells. Proteins were quantified using a microplate reader and separated by centrifugation. Protein suspensions (15 $\mathrm{mg}$ protein) were electrophoresed and transferred to a nitrocellulose membrane, which was then blocked with 5\% skim milk. The membrane was exposed to anti-Bcl-2 primary antibody $(1: 800)$ at $4{ }^{\circ} \mathrm{C}$ for $12 \mathrm{~h}$ and anti-mouse immunoglobulin $\mathrm{G}$ secondary antibody (1:1500) at $37^{\circ} \mathrm{C}$ for $2 \mathrm{~h}$, being washed three times after each incubation. Horseradish peroxidase activity was used to visualize specific protein bands with a gel imaging system. Protein quantification was based on grayscale intensity.

\section{Examination of caspase-3 activity}

Caspase-3 activity was tested with a caspase-3 kit (Song et al., 2015). Transfected $\mathrm{SiHa}$ cells $\left(1 \times 10^{5}\right)$ were treated with a chromogenic substrate on a six-well plate and incubated at room temperature for $20 \mathrm{~min}$. The chromophore p-nitroaniline was then detected by light absorbance using a microplate reader (Thermo Scientific, Waltham, MA, USA). All experiments were repeated three times. Relative caspase-3 activity was estimated with a routine protocol (Song et al., 2015).

\section{Alteration of $\mathrm{Bcl}-2$ expression}

Bcl-2 siRNA and expression plasmid (at a final concentration of $10 \mathrm{nM}$ ) (pcDNA3.1 with $\mathrm{CMV}$ as the promoter) were transfected separately into SiHa cells using Lipofectamine 2000. miR-187 or control miRNA (at a final concentration of $10 \mathrm{nM}$ ) were then transfected into the same cells.

\section{Statistical analysis}

The SPSS 14.0 software (SPSS Inc., Chicago, IL, USA) was used for data analysis. Normally distributed measurement data are reported as means \pm standard deviations. The $t$-test was performed to assess statistical significance, with $\mathrm{P}$ values $<0.05$ being considered statistically significant. All experiments were repeated three times.

Genetics and Molecular Research 16 (1): gmr16018969 


\section{RESULTS}

\section{miR-187 transfection decreased cell viability and reduced cell growth}

Compared to control miRNA, transfection with miR-187 significantly decreased the viability of SiHa cells, as measured by MTT assays $(\mathrm{P}<0.01)$. No difference was observed between untransfected SiHa cells and those treated with control miRNA (Figure 1; P > 0.05). Therefore, the latter were used as a negative control in subsequent experiments.

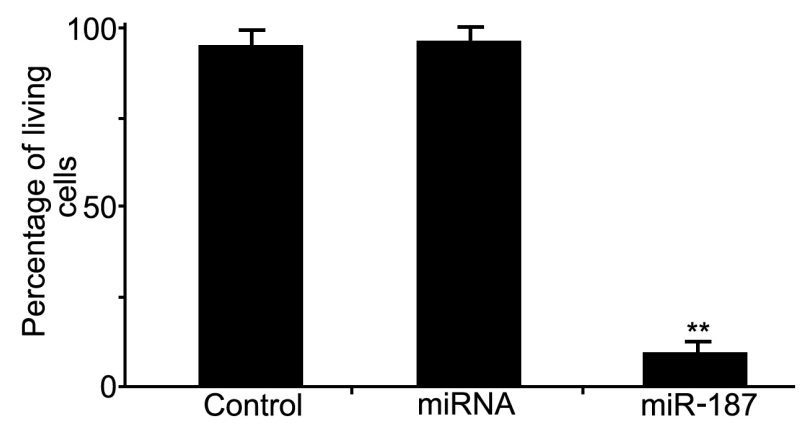

Figure 1. Analysis of cell viability in three treatment groups. ${ }^{*} * \mathrm{P}<0.01$ versus control microRNA (miRNA).

\section{miR-187 transfection induced apoptosis of SiHa cells}

miR-187 transfection significantly increased phosphatidylserine translocation compared to the control miRNA according to annexin V-FITC double staining (Figure 2; $\mathrm{P}=$ 0.0031). This suggests that apoptosis was induced by miR-187.

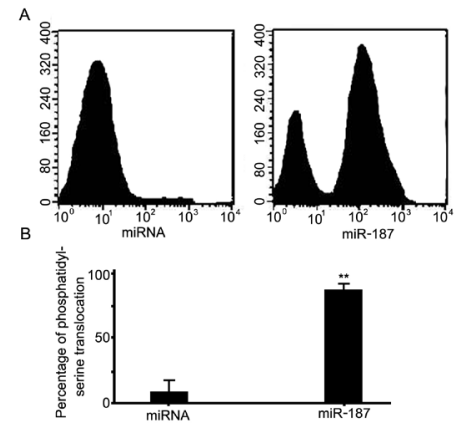

Figure 2. miR-187 transfection enhanced translocation of phosphatidylserine. $* * \mathrm{P}<0.01$ versus control microRNA (miRNA). A. A representative histogram of annexin-V expression (phosphatidylserine translocation) analyzed by flow cytometry. B. Percentage of phosphatidylserine translocation in SiHa cells transfected with miRNA or miR-187.

\section{miR-187 transfection activated caspase-3 in SiHa cells}

Compared with the control miRNA construct, miR-187 transfection significantly increased caspase-3 activity in $\mathrm{SiHa}$ cells (Figure 3; $\mathrm{P}<0.05$ ), implying that this miRNA promoted apoptosis via activation of this enzyme. 


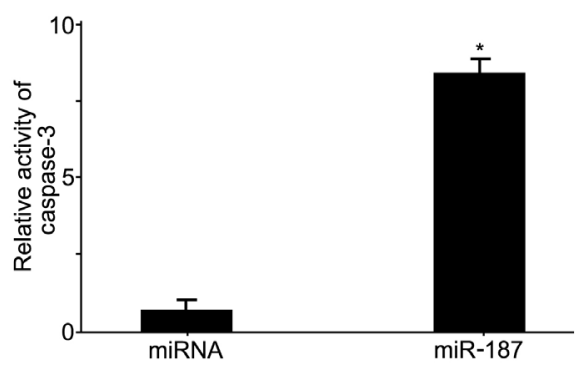

Figure 3. miR-187 transfection increased caspase- 3 activity in SiHa cells. $* \mathrm{P}<0.05$ versus control microRNA (miRNA).

\section{miR-187 transfection decreased Bcl-2 protein expression in SiHa cells}

In comparison to SiHa cells transfected with control miRNA, those treated with miR187 exhibited significantly decreased expression of Bcl-2 protein (Figure 4), as observed by western blotting.

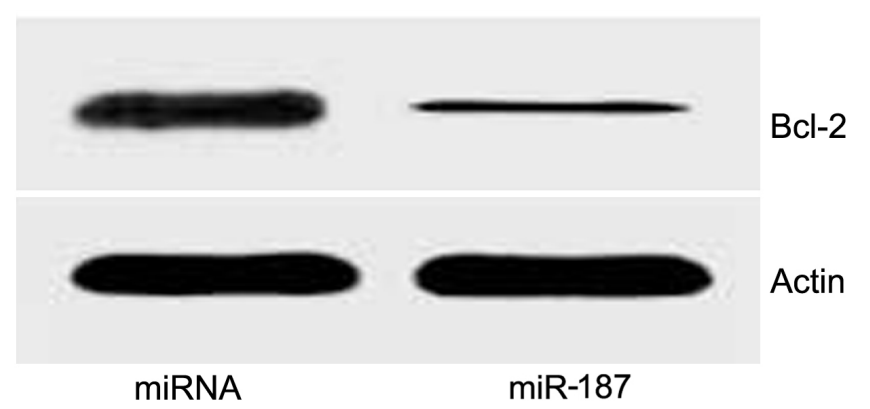

Figure 4. Specific protein bands detected by western blot. miR-187 transfection decreased Bcl-2 protein expression in $\mathrm{SiHa}$ cells. miRNA = control microRNA.

\section{Bcl-2 inhibition enhanced the pro-apoptotic effect of miR-187}

As shown in Figure 5A, Bcl-2 siRNA successfully inhibited expression of its target, and enhanced the decrease in Bcl-2 levels induced by miR-187. Moreover, the Bcl-2 siRNA+miR-187 group demonstrated the highest levels of caspase-3 activity (Figure 5B), suggesting that Bcl-2 inhibition increased the pro-apoptotic effect of miR-187. However, other BCL-2 independent effects might also be involved in miR-187-mediated cell apoptosis as miR-187 also has BCL-2-independent activities.

\section{Bcl-2 overexpression negated the pro-apoptotic effect of miR-187}

As shown in Figure 6A, the Bcl-2 plasmid effectively elevated Bcl-2 expression, and attenuated the reduction of this protein caused by miR-187. In addition, the Bcl2 plasmid + miR-187 group exhibited lower caspase-3 activity than the miR-187 group (Figure 6B). This indicates that $\mathrm{Bcl}-2$ overexpression diminished the pro-apoptotic influence of miR-187.

Genetics and Molecular Research 16 (1): gmr16018969 
A
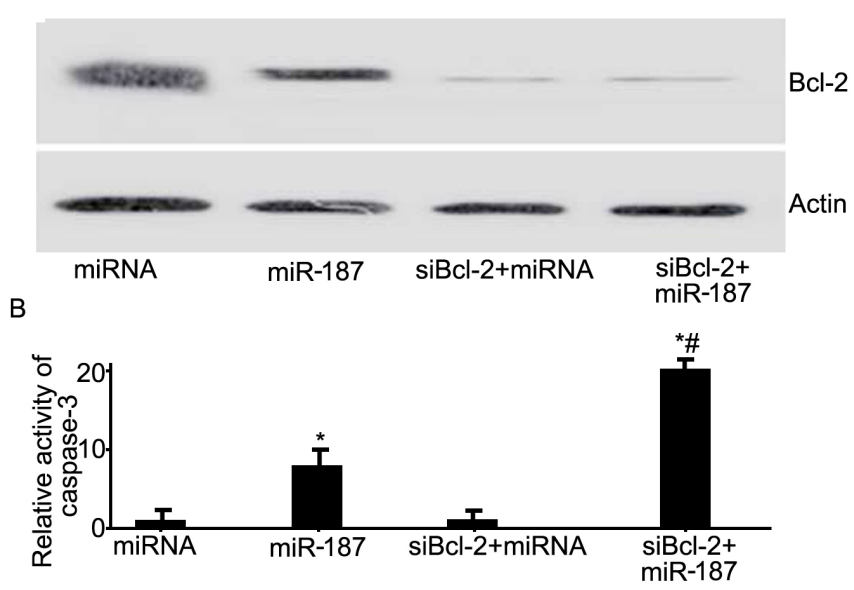

Figure 5. A. Western blotting results for each treatment group. B. Analysis of caspase- 3 activity in each group. $* \mathrm{P}$ $<0.05$ versus control microRNA (miRNA). ${ }^{*} \mathrm{P}<0.05$ for Bcl- 2 small interfering RNA (siBcl-2)+miR-187 versus miR-187.
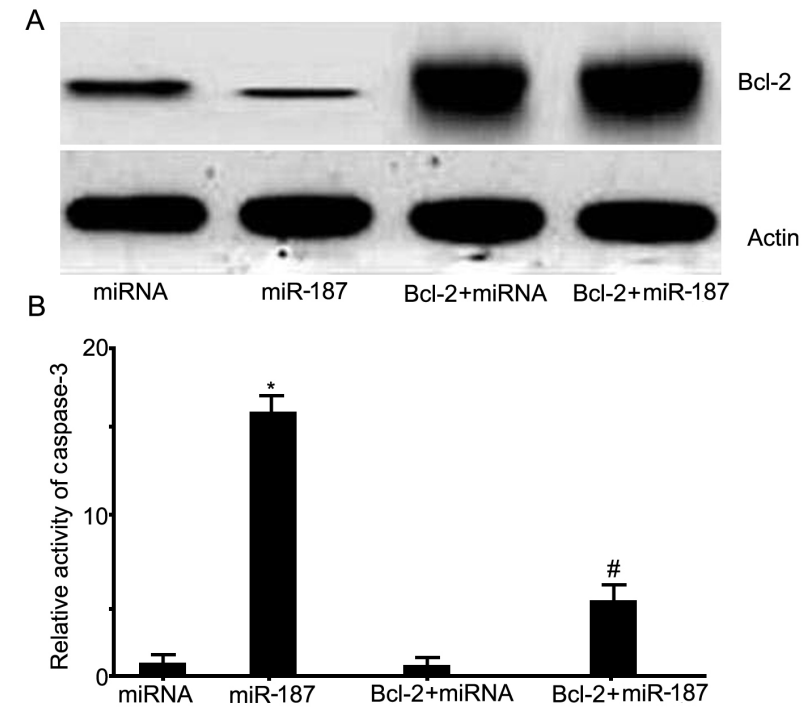

Figure 6. A. Western blotting results for each treatment group. B. Analysis of caspase-3 activity in each group. *P $<0.05$ versus control microRNA (miRNA). ${ }^{\mathrm{P}}<0.05$ for $\mathrm{Bcl}-2$ expression plasmid (Bcl-2) + miR-187 versus miR187. Differences that can account for BCL-2 are not being targeted by the miRNA.

\section{DISCUSSION}

Our study explored the effect of miR-187 on cervical cancer in a SiHa cell model. Furthermore, specific mechanisms were identified, furthering our understanding of its role in this malignancy. We found that miR-187 reduced the viability of cervical cancer cells, 
inhibited their growth, and induced their apoptosis, consistent with the findings of a previous study (Pedroza-Torres et al., 2014).

Prior research has offered limited data concerning the mechanisms underlying the effect of miR-187 on cervical cancer (Pedroza-Torres et al., 2014). Recent study has demonstrated that miR-218 significantly inhibits cervical cancer growth, and miR-34a is associated with tumor metastasis (Xu et al., 2013; Li et al., 2015). This implies that miRNAs are involved in the progression of cervical cancer.

Bcl-2 is an anti-apoptosis protein widely expressed in different cell types (Lin et al., 2015), but its status as a target regulated by miR-187 remains unclear. In addition, the specific mechanisms responsible for the functions of Bcl-2 warrant further investigation (Bhattacharjya et al., 2015; Honegger et al., 2015). The present study revealed that miR-187 does indeed decrease Bcl-2 expression. Moreover, apoptosis of $\mathrm{SiHa}$ cells was enhanced as a result of miR187-induced Bcl-2 inhibition. Furthermore, overexpression of Bcl-2 negated the pro-apoptotic effect of miR-187. Bcl-2 is recognized as an inhibitor of apoptosis. This study explored its impact on tumor progression through overexpression and RNA interference.

There were three key findings in the present investigation that demonstrate the pivotal role played by Bcl-2 in the pro-apoptotic effect of miR-187 on SiHa cells: 1) Bcl2 protein expression was significantly reduced in SiHa cells transfected with miR-187;2) Bcl-2 siRNA enhanced the pro-apoptotic influence of miR-187; and 3) plasmid-based Bcl2 overexpression attenuated apoptosis induced by miR-187. Together, these observations suggest that miR-187 affects apoptosis of SiHa cells via downregulation of Bcl-2. Previous studies have shown Bcl-2 to be involved in other cancers, enhancing cancer cell proliferation and exacerbating lesions (Lao et al., 2014; Geng et al., 2015; Kogo et al., 2015). However, none of these have addressed the relationship between Bcl-2 and cervical cancer. Our study is the first to demonstrate that Bcl-2 also inhibits apoptosis in cervical cancer, identifying it as a potential target for molecular therapy based on regulation by miR-187 (Zhou and Wang, 2015). However, we cannot exclude other BCL-2 independent effects during miR187-mediated cell apoptosis and requires further investigation.

Three limitations to our study should be considered: 1) we did not test tumor tissue and adjacent normal tissue from cervical cancer patients; 2) our study did not assess long-term prognosis in relation to Bcl-2 expression; and 3) animal models are needed to explore the effect of miR-187 on cervical cancer in vivo.

\section{CONCLUSION}

miR-187 induces apoptosis of cervical cancer cells via downregulation of Bcl-2. Bcl2 is a potential drug target for treatment of this disease, and its inhibition could improve the efficacy of clinical therapy.

\section{Conflicts of interest}

The authors declare no conflict of interest.

\section{ACKNOWLEDGMENTS}

We thank the anonymous reviewers for reviewing this manuscript.

Genetics and Molecular Research 16 (1): gmr16018969 
miR-187-induced cervical carcinoma cell apoptosis

\section{REFERENCES}

Bhattacharjya S, Roy KS, Ganguly A, Sarkar S, et al. (2015). Inhibition of nucleoporin member Nup214 expression by miR-133b perturbs mitotic timing and leads to cell death. Mol. Cancer 14: 42. http://dx.doi.org/10.1186/s12943-015$\underline{0299-\mathrm{Z}}$

Bonetta AC, Mailly L, Robinet E, Travé G, et al. (2015). Artificial microRNAs against the viral E6 protein provoke apoptosis in HPV positive cancer cells. Biochem. Biophys. Res. Commun. 465: 658-664. http://dx.doi.org/10.1016/j. bbrc.2015.07.144

Bumrungthai S, Ekalaksananan T, Evans MF, Chopjitt P, et al. (2015). Up-regulation of miR-21 is associated with cervicitis and human papillomavirus infection in cervical tissues. PLoS One 10: e0127109. http://dx.doi.org/10.1371/journal. pone. 0127109

Chang YW, Chen MW, Chiu CF, Hong CC, et al. (2014). Arsenic trioxide inhibits CXCR4-mediated metastasis by interfering miR-520h/PP2A/NF-kB signaling in cervical cancer. Ann. Surg. Oncol. 21 (Suppl 4): S687-S695. http:// dx.doi.org/10.1245/s10434-014-3812-5

Chen Y, Ke G, Han D, Liang S, et al. (2014). MicroRNA-181a enhances the chemoresistance of human cervical squamous cell carcinoma to cisplatin by targeting PRKCD. Exp. Cell Res. 320: 12-20. http://dx.doi.org/10.1016/j. yexcr.2013.10.014

Cong J, Liu R, Wang X, Jiang H, et al. (2016). MiR-634 decreases cell proliferation and induces apoptosis by targeting mTOR signaling pathway in cervical cancer cells. Artif. Cells Nanomed. Biotechnol. 44: 1694-1701. http://dx.doi.or $\mathrm{g} / 10.3109 / 21691401.2015 .1080171$

Deng B, Zhang Y, Zhang S, Wen F, et al. (2015). MicroRNA-142-3p inhibits cell proliferation and invasion of cervical cancer cells by targeting FZD7. Tumour Biol. 36: 8065-8073. http://dx.doi.org/10.1007/s13277-015-3483-2

Geng D, Song X, Ning F, Song Q, et al. (2015). MiR-34a inhibits viability and invasion of human papillomavirus-positive cervical cancer cells by targeting E2F3 and regulating survivin. Int. J. Gynecol. Cancer 25: 707-713. http://dx.doi. org $/ 10.1097 /$ IGC. 0000000000000399

Honegger A, Schilling D, Bastian S, Sponagel J, et al. (2015). Dependence of intracellular and exosomal microRNAs on viral E6/E7 oncogene expression in HPV-positive tumor cells. PLoS Pathog. 11: e1004712. http://dx.doi.org/10.1371/ journal.ppat.1004712

Huang F, Lin C, Shi YH and Kuerban G (2013). MicroRNA-101 inhibits cell proliferation, invasion, and promotes apoptosis by regulating cyclooxygenase-2 in Hela cervical carcinoma cells. Asian Pac. J. Cancer Prev. 14: 59155920. http://dx.doi.org/10.7314/APJCP.2013.14.10.5915

Jia LF, Gan YH and Yu GY (2016). [Relationships between microRNA expressions and prognosis in patients with tongue squamous cell carcinoma and the mechanisms microRNA regulating tongue squamous cell carcinoma biological behavior]. Beijing Da Xue Xue Bao 48: 5-9.

Kogo R, How C, Chaudary N, Bruce J, et al. (2015). The microRNA-218 Survivin axis regulates migration, invasion, and lymph node metastasis in cervical cancer. Oncotarget 6: 1090-1100. http://dx.doi.org/10.18632/oncotarget.2836

Kong Q, Wang W and Li P (2015). Regulator role of HPV E7 protein on miR-21 expression in cervical carcinoma cells and its functional implication. Int. J. Clin. Exp. Pathol. 8: 15808-15813.

Lao G, Liu P, Wu Q, Zhang W, et al. (2014). Mir-155 promotes cervical cancer cell proliferation through suppression of its target gene LKB1. Tumour Biol. 35: 11933-11938. http://dx.doi.org/10.1007/s13277-014-2479-7

Li J, Hu L, Tian C, Lu F, et al. (2015). microRNA-150 promotes cervical cancer cell growth and survival by targeting FOXO4. BMC Mol. Biol. 16: 24. http://dx.doi.org/10.1186/s12867-015-0052-6

Lin L, Cai Q, Zhang X, Zhang H, et al. (2015). Two less common human microRNAs miR-875 and miR-3144 target a conserved site of E6 oncogene in most high-risk human papillomavirus subtypes. Protein Cell 6: 575-588. http:// dx.doi.org/10.1007/s13238-015-0142-8

Liu S, Zhang P, Chen Z, Liu M, et al. (2013). MicroRNA-7 downregulates XIAP expression to suppress cell growth and promote apoptosis in cervical cancer cells. FEBS Lett. 587:2247-2253. http://dx.doi.org/10.1016/j.febslet.2013.05.054

Liu S, Pan X, Yang Q, Wen L, et al. (2015). MicroRNA-18a enhances the radiosensitivity of cervical cancer cells by promoting radiation-induced apoptosis. Oncol. Rep. 33: 2853-2862.

Luo M, Shen D, Zhou X, Chen X, et al. (2013). MicroRNA-497 is a potential prognostic marker in human cervical cancer and functions as a tumor suppressor by targeting the insulin-like growth factor 1 receptor. Surgery 153: 836-847. http://dx.doi.org/10.1016/j.surg.2012.12.004

Pedroza-Torres A, López-Urrutia E, García-Castillo V, Jacobo-Herrera N, et al. (2014). MicroRNAs in cervical cancer: evidences for a miRNA profile deregulated by HPV and its impact on radio-resistance. Molecules 19: 6263-6281. http://dx.doi.org/10.3390/molecules 19056263

Genetics and Molecular Research 16 (1): gmr16018969 
Phuah NH, In LL, Azmi MN, Ibrahim H, et al. (2013). Alterations of microRNA expression patterns in human cervical carcinoma cells (Ca Ski) toward 1'S-1'-acetoxychavicol acetate and cisplatin. Reprod. Sci. 20: 567-578. http:// dx.doi.org/10.1177/1933719112459220

Song L, Liu S, Zeng S, Zhang L, et al. (2015). miR-375 modulates radiosensitivity of HR-HPV-positive cervical cancer cells by targeting UBE3A through the p53 pathway. Med. Sci. Monit. 21: 2210-2217. http://dx.doi.org/10.12659/ MSM.893859

Wen SY, Lin Y, Yu YQ, Cao SJ, et al. (2015). miR-506 acts as a tumor suppressor by directly targeting the hedgehog pathway transcription factor Gli3 in human cervical cancer. Oncogene 34: 717-725. http://dx.doi.org/10.1038/ onc.2014.9

Xu J, Li Y, Wang F, Wang X, et al. (2013). Suppressed miR-424 expression via upregulation of target gene Chkl contributes to the progression of cervical cancer. Oncogene 32: 976-987. http://dx.doi.org/10.1038/onc.2012.121

Yang L, Wang YL, Liu S, Zhang PP, et al. (2014). miR-181b promotes cell proliferation and reduces apoptosis by repressing the expression of adenylyl cyclase 9 (AC9) in cervical cancer cells. FEBS Lett. 588: 124-130. http:// dx.doi.org/10.1016/j.febslet.2013.11.019

Yu Q, Liu SL, Wang H, Shi G, et al. (2014). miR-126 Suppresses the proliferation of cervical cancer cells and alters cell sensitivity to the chemotherapeutic drug bleomycin. Asian Pac. J. Cancer Prev. 14: 6569-6572. http://dx.doi. org/10.7314/APJCP.2013.14.11.6569

Yuan W, Xiaoyun H, Haifeng Q, Jing L, et al. (2014). MicroRNA-218 enhances the radiosensitivity of human cervical cancer via promoting radiation induced apoptosis. Int. J. Med. Sci. 11: 691-696. http://dx.doi.org/10.7150/ijms.8880

Zhang J, Li S, Yan Q, Chen X, et al. (2013). Interferon- $\beta$ induced microRNA-129-5p down-regulates HPV-18 E6 and E7 viral gene expression by targeting SP1 in cervical cancer cells. PLoS One 8: e81366. http://dx.doi.org/10.1371/ journal.pone. 0081366

Zhou XL and Wang M (2015). Expression levels of survivin, Bcl-2, and KAI1 proteins in cervical cancer and their correlation with metastasis. Genet. Mol. Res. 14: 17059-17067. http://dx.doi.org/10.4238/2015.December.16.6

Genetics and Molecular Research 16 (1): gmr16018969 